\title{
Laparoscopic loop duodenaljejunal bypass with sleeve gastrectomy in type 2 diabetic patients
}

\begin{abstract}
Background: Laparoscopic loop duodenojejunal bypass with sleeve gastrectomy (LDJB-SG) is a new metabolic procedure. Our initial data on type 2 diabetes (T2D) remission after LDJB-SG were promising. Objectives: The aim of this study was to look at our intermediate outcomes after LDJB-SG. Setting: An academic medical center. Methods: A prospective analysis of T2D patients who underwent LDJB-SG between October 2011 and October 2014 was performed. Data collected included baseline demographic, body mass index, fasting blood glucose, glycosylated hemoglobin, C-peptide, resolution of co-morbidities, and postoperative complications. Results: A total of 163 patients with minimum of follow-up >1 year were enrolled in this study (57 men and 106 women). The mean age and body mass index were $47.7( \pm 10.7)$ years and a $30.2( \pm 5.1) \mathrm{kg} / \mathrm{m} 2$, respectively. There were 119 patients on oral hypoglycemic agents only, 29 patients were on oral hypoglycemic agents and insulin, 3 patients were on insulin only, and the other 12 patients were not on diabetic medication. Mean operation time and length of hospital stay were $144.7( \pm 45.1)$ minutes and $2.4( \pm 1.0)$ days, respectively. Seven patients $(3.6 \%)$ needed reoperation due to bleeding $(\mathrm{n}=1)$, anastomotic leak $(n=2)$, sleeve strictures $(n=2)$, and incisional hernia $(n=2)$. At 2 years of follow-up, there were 56 patients. None of the patients were on insulin and only $20 \%$ of patients were on oral hypoglycemic agents. Mean body mass index significantly dropped to $22.9( \pm 5.6) \mathrm{kg} / \mathrm{m} 2$ at 2 years. The mean preoperative fasting blood glucose, glycosylated hemoglobin, and C-peptide levels were $174.7 \mathrm{mg} / \mathrm{dL}( \pm 61.0), 8.8 \%( \pm 1.8)$, and $2.6( \pm 1.7)$ $\mathrm{ng} / \mathrm{mL}$, respectively. The mean fasting blood glucose, glycosylated hemoglobin, and Cpeptide at 2 years were $112.5( \pm 60.7) \mathrm{mg} / \mathrm{dL}, 6.4 \%( \pm 2.0)$, and $1.5( \pm 0.6) \mathrm{ng} / \mathrm{mL}$, respectively. No patient needed revisional surgery because of dumping syndrome, marginal ulcer, or gastroesophageal reflux disease at the last follow up period. Conclusion: At 2 years, LDJB-SG is a relatively safe and effective metabolic surgery with significant weight loss and resolution of co-morbidities.
\end{abstract}

Keyword: Complication; Diabetes; Duodenojejunal bypass; Metabolic surgery; Obesity; Sleeve gastrectomy; Weight loss 